\title{
Desafios para o cuidado farmacêutico na Atenção Primária à Saúde
}

\author{
$1{ }^{1}$ Délcia Regina Destro, ${ }^{2}$ Simone Alves do Vale, ${ }^{3}$ Maria José Menezes Brito, \\ ${ }^{4}$ Clarice Chemello I
}

Resumo: Este estudo de caso objetivou descrever o perfil dos farmacêuticos, caracterizar os serviços farmacêuticos e desvelar os fatores determinantes para a provisão do acompanhamento farmacoterapêutico fundamentados no modelo de prática do cuidado farmacêutico na Atenção Primária à Saúde. Cinquenta farmacêuticos responderam a um questionário sobre seu perfil e atividades. Aplicou-se a análise de conteúdo de Bardin para análise dos dados sobre as atividades, resultando em duas categorias: caracterização das atividades desempenhadas pelos farmacêuticos e os determinantes para a provisão do acompanhamento farmacoterapêutico. Observou-se que o cuidado farmacêutico ainda é um desafio a ser enfrentado, principalmente devido à demanda de atividades gerenciais e à deficiência na formaçáo para o cuidado, necessitando reorganizar os processos de trabalho e as diretrizes institucionais para a ampliação do acesso aos serviços farmacêuticos centrados no paciente. Destaca-se, contudo, o crescente número de farmacêuticos trabalhando no cuidado, reorganizando e redefinindo seu papel, buscando transformar a realidade da Assistência Farmacêutica municipal. Apesar dos desafios ainda existentes referentes a formação, estrutura e processos, destaca-se a busca de soluçóes para a organização do serviço, com o aprimoramento das atividades clínicas, fortalecendo a Assistência Farmacêutica no Sistema Único de Saúde.

> Palavras-chave: cuidado farmacêutico; serviços farmacêuticos; Atenção Básica em Saúde; Sistema Único de Saúde.

\author{
'Faculdade de Farmácia, \\ Universidade Federal de Minas \\ Gerais / Secretaria Municipal \\ de Saúde de Belo Horizonte. \\ Belo Horizonte-MG, Brasil \\ (delciadestro@gmail.com). \\ ORCID: 0000-0003-1058-1374 \\ 2 Secretaria Municipal de \\ Saúde de Belo Horizonte. Belo \\ Horizonte-MG, Brasil (simone. \\ vale@pbh.gov.br). \\ ORCID: 0000-0002-4160-8878 \\ ${ }^{3}$ Faculdade de Enfermagem, \\ Universidade Federal de Minas \\ Gerais. Belo Horizonte-MG, Brasil \\ (mj.brito@globo.com. \\ ORCID: 0000-0001-9183-1982 \\ ${ }^{4}$ Faculdade de Farmácia, \\ Universidade Federal de Minas \\ Gerais. Belo Horizonte-MG, Brasil \\ (clachemello@gmail.com). \\ ORCID: 0000-0002-1234-1561
}

Recebido em: 11/05/2019 Aprovado em: 03/03/2020 Revisado em: 27/07/2021 


\section{Introdução}

O cuidado farmacêutico, termo adotado recentemente pelo Ministério da Saúde (MS) como sinônimo de Atenção Farmacêutica, consiste na "ação integrada do farmacêutico com a equipe de saúde, centrada no usuário, para promoção, proteção e recuperação da saúde e prevenção de agravos". Visa à educação em saúde e a promoção do Uso Racional de Medicamentos (URM) prescritos e não prescritos, por meio dos serviços da clínica farmacêutica e atividades técnico-pedagógicas, voltadas ao indivíduo, à família, à comunidade (IFC) e à equipe de saúde, baseados em uma relação terapêutica entre farmacêutico e paciente e de corresponsabilização pelas necessidades farmacoterapêuticas dos pacientes (CFF, 2016; BRASIL, 2014). A filosofia do Cuidado Farmacêutico atribui a este profissional a responsabilidade de garantir que os pacientes recebam a terapia mais indicada, efetiva, segura e conveniente (WIEDENMAYER et al., 2006). E é no acompanhamento farmacoterapêutico (AFT), um dos serviços farmacêuticos, que o farmacêutico assume essa responsabilidade (BRASIL, 2018).

Diante das demandas de saúde atuais da população brasileira - envelhecimento da população, elevado uso de medicamentos, baixa adesão a tratamentos e desarticulação das práticas profissionais - é imperativo aos profissionais de saúde, em particular o farmacêutico, a necessidade de avançar na qualificação do cuidado ofertado aos usuários de medicamentos (COSTA et al., 2017a). A estruturação da Assistência Farmacêutica (AF) no Sistema Único de Saúde (SUS) do Brasil consiste em importante estratégia para a ampliação e qualificação do acesso da população aos medicamentos essenciais e ao Cuidado Farmacêutico. O farmacêutico no Núcleo de Apoio a Saúde da Família (Nasf) atua tanto na atenção ao cuidado dos indivíduos, participando de forma ativa de sua terapêutica, como nas atividades gerenciais, em relação à gestão dos medicamentos das farmácias dos Centros de Saúde (CS) (BRASIL, 2018).

Neste contexto, as atribuiçôes do farmacêutico no SUS dividem-se em técnico gerenciais e assistenciais. A primeira compreende as atividades de seleção, programação, aquisição, armazenamento e distribuição de medicamentos de forma integrada para garantir o acesso aos medicamentos (CORRER; OTUKI; SOLER, 2011; BRASIL, 2014). As atividades assistenciais visam qualificar a atenção integral aos usuários, e, também, potencializar açôes realizadas pelos demais profissionais relacionadas ao URM (BRASIL, 2014). Essas atividades serão operacionalizadas por 
meio de serviços farmacêuticos, fundamentados pela filosofia de prática do Cuidado Farmacêutico (CFF, 2016).

No que tange à Atenção Primária à Saúde (APS) cujas ações são desenvolvidas nos Centros de Saúde (CS), o farmacêutico pode estar inserido no Nasf, para apoiar a inserção da Estratégia de Saúde da Família (ESF) na rede de serviços e ampliar a abrangência, resolutividade, territorialização, regionalização e ações da APS (BRASIL, 2018). No município de Belo Horizonte (BH), as 82 equipes do Nasf contam com um farmacêutico, como provedor dos serviços farmacêuticos e das atividades técnico-gerenciais, em relação à gestão dos medicamentos das farmácias dos CS (BELO HORIZONTE, 2018).

Em consonância com a Organização Pan-Americana da Saúde (OPAS), o Ministério da Saúde e o Conselho Federal de Farmácia, a Secretaria Municipal de Saúde de Belo Horizonte (SMSA-BH) trabalha para ampliar as açóes do farmacêutico de forma integrada, objetivando o cuidado integral do usuário, além do acesso aos medicamentos de maneira contínua e regular (BELO HORIZONTE, 2018; BRASIL, 2018; CFF, 2016; BRASIL, 2014; OPAS, 2013).

A inserção do farmacêutico na APS, com perspectivas de fortalecimento da sua integração à equipe, tem sido impulsionada pelas recentes mudanças institucionais e normativas no cenário nacional, sendo um processo em construção (BARBERATO; SCHERER; LACOURT, 2018). Diante do exposto, os objetivos do presente trabalho foram descrever o perfil dos farmacêuticos, caracterizar os serviços farmacêuticos e desvelar os fatores determinantes para a provisão do AFT fundamentados no modelo de prática do Cuidado Farmacêutico na APS em BH.

\section{Metodologia}

Trata-se de estudo de caso, descritivo e interpretativo, realizado em maio de 2016, em Belo Horizonte, Minas Gerais, Brasil (YIN, 2015; MINAYO, 2014). Foram convidados a participar da pesquisa todos os farmacêuticos atuantes, há, pelo menos, um ano, nos CS da APS/Nasf da SMSA-BH (64 farmacêuticos), considerando que nesse município todos os farmacêuticos da APS encontram-se vinculados às equipes do Nasf. Os dados foram coletados por meio de um questionário, abrangendo questôes abertas e fechadas relativas ao perfil sociodemográfico, funcional e formativo dos farmacêuticos, e às atividades desempenhadas. Nesse município, a institucionalização do Nasf em 2008, propiciou efetivamente a inserção do 
farmacêutico na APS e apresentou aumento progressivo do número de profissionais, proporcional à ampliação das equipes do Nasf. Atualmente, todos os $82 \mathrm{Nasf}$ em $\mathrm{BH}$ contam com um farmacêutico, que desenvolve as atividades de AF.

Os dados sociodemográficos, funcional e formativo dos farmacêuticos foram analisados utilizando-se o programa SPSS para Windows versão 16.0, sendo apresentados de forma descritiva (média \pm desvio padrão e frequência $-\%$ ).

Analisaram-se as respostas às questôes abertas pela análise de conteúdo de Bardin (2016), a qual compreende 3 etapas: 1) Pré-análise - transcrição, na íntegra, leitura flutuante e exaustiva; 2) Exploração do material - consiste na transformação dos dados brutos, quando surgem os temas mais relevantes definindo as categorias temáticas; 3) Tratamento dos resultados obtidos e interpretação, quando podemos propor inferências e adiantar interpretaçóes a propósito dos objetivos previstos ou que digam respeito a outras descobertas inesperadas.

Este trabalho faz parte de um trabalho de doutorado, foi aprovado pelos Comitês de Ética da SMSA-BH e Universidade Federal de Minas Gerais. Todos os participantes assinaram o Termo de Consentimento Livre e Esclarecido.

\section{Resultados e Discussão}

Dos 64 farmacêuticos atuantes nos Nasf/APS da SMSA-BH no momento da pesquisa, $50(78 \%)$ responderam ao questionário. Os resultados desta pesquisa estão divididos em duas partes: a primeira, dados descritivos relativos ao perfil sociodemográfico, funcional e formativo dos farmacêuticos e a segunda, aborda a análise de conteúdo das questôes abertas, configuradas em categorias: caracterização das atividades desempenhadas pelos farmacêuticos e os determinantes para a provisão do AFT.

\section{Perfil sociodemográfico, funcional e formativo dos farmacêuticos}

Dos 50 farmacêuticos, $80 \%$ eram mulheres, média de idade de 35,8 anos e $60 \%$ casados, resultado semelhante ao relatório do CFF sobre o perfil do farmacêutico no Brasil (CFF, 2015). Cada farmacêutico era designado como coordenador técnico por 2,4 CS, em média, sendo que $56 \%$ deles por até 2 CS, onde atuava sua equipe do Nasf.

Quarenta e um farmacêuticos trabalhavam na SMSA-BH há menos de 5 anos (82\%), média de 4,2 anos. O tempo médio na função foi de 3,4 anos, todos possuíam carga horária de 40 horas semanais e $76 \%$ efetivos (tabela 1). 
Tabela 1. Perfil sociodemográfico dos farmacêuticos da Atenção Primária à Saúde da Secretaria Municipal da Saúde de Belo Horizonte (SMSA-BH), 2016

\begin{tabular}{lll}
\hline Variável & n $(50)$ & $\%$ \\
\hline
\end{tabular}

Gênero

$\begin{array}{lll}\text { Feminino } & 40 & 80 \\ \text { Masculino } & 10 & 20\end{array}$

Faixa etária

$\begin{array}{lll}\text { 19-28 anos } & 2 & 4 \\ 29-38 \text { anos } & 36 & 72 \\ 37-48 \text { anos } & 7 & 14 \\ 49-58 \text { anos } & 4 & 4 \\ 59 \text { a } 68 \text { anos } & 3 & 6\end{array}$

Estado civil

$\begin{array}{lll}\text { Solteiro } & 15 & 30 \\ \text { Casado } & 30 & 60 \\ \text { Outros } & 5 & 10\end{array}$

Tempo na SMSA-BH

$\begin{array}{lll}\text { Até } 5 \text { anos } & 41 & 82 \\ 6 \text { a } 10 \text { anos } & 8 & 16 \\ >10 \text { anos } & 1 & 2\end{array}$

Tempo na função

$\begin{array}{lll}\text { Até } 2 \text { anos } & 9 & 18 \\ 2,1 \text { a } 4 \text { anos } & 32 & 64 \\ 4,1 \text { a } 6 \text { anos } & 7 & 14 \\ >6 \text { anos } & 2 & 4\end{array}$

Vínculo

\begin{tabular}{lll} 
Efetivo SMSA-BH & 28 & 76 \\
Contrato administrativo & 12 & 24 \\
\hline
\end{tabular}

Fonte: Dados da pesquisa. 
Em relação à formação acadêmica, a maioria (76\%) graduou-se em instituiçôes públicas, sendo que $80 \%$ concluiu a graduação depois de 2002 . A formação acadêmica era diversificada, sendo $40 \%$ indústria, 32\% sem habilitação, $22 \%$ análises clínicas, $4 \%$ alimentos e $2 \%$ não informado. Do total, 41 (82\%) cursaram pós-graduação, sendo $71 \%$ especialização, $23 \%$ mestrado e $6 \%$ residência (tabela 2 ).

Tabela 2. Formação dos profissionais farmacêuticos da Atenção Primária à Saúde da Secretaria Municipal da Saúde de Belo Horizonte (SMSA-BH), SMSA-BH, 2016

\begin{tabular}{lll}
\hline Variáveis & $\mathrm{N}(50)$ & $\%$ \\
\hline
\end{tabular}

Tipo de instituição de ensino (50)

$\begin{array}{lll}\text { Pública } & 38 & 76 \\ \text { Privada } & 12 & 24\end{array}$

Tempo de graduação

Até 2002

Depois de 2002

Habilitação

\begin{tabular}{lll} 
Análises clínicas & 11 & 22 \\
Indústria & 20 & 40 \\
Alimentos & 2 & 4 \\
Não informou a área & 1 & 2 \\
Sem habilitação & 16 & 32 \\
Especialização & & \\
Residência & 37 & 71,2 \\
Mestrado & 3 & 5,8 \\
& 12 & 23,1 \\
Sim & & \\
Não & 15 & 30 \\
Sim & 35 & 70 \\
Não & 21 & 42 \\
\hline
\end{tabular}

Pós-graduação*

$10 \quad 20$

$40 \quad 80$


Entre aqueles com especialização, 46\% foi em área clínica. Sobre a experiência em Atenção Farmacêutica, 30\% respondeu ter experiência formativa (cursado disciplinas na área, ter sido bolsista de iniciação científica ou de extensão, participação em grupos de estudo) e 42\%, experiência profissional. A experiência formativa mais citada (52\%) foi a participação no Grupo de Estudos em Atenção Farmacêutica em universidade e, entre as experiências profissionais, 71\% referenciaram o trabalho na SMSA-BH.

As Diretrizes Curriculares Nacionais (DCN) para o curso de Farmácia de 2002 redirecionaram o foco de formação do medicamento para o processo saúde-doença e sua atuação no SUS, em resposta à demanda social (SOUZA; BASTOS; BOGO, 2013; BRASIL, 2002). Entretanto, a formação do farmacêutico em relação à atenção à saúde ainda é deficitária, pois a forma tecnicista e fragmentada, enfatizando o modelo biomédico, deixa lacunas no conhecimento, nas habilidades e atitudes clínicas e humanísticas (CASTRO, 2011). Em 2017 foram aprovadas novas DCN, as quais direcionam os currículos para a formação de profissional de saúde generalista, voltado prioritariamente ao cuidado e ao SUS. O egresso deverá ter competências que atendam às necessidades de saúde da população, representando um grande avanço, seguindo a tendência mundial da profissão: o cuidado centrado no paciente (BRASIL, 2017; FEDERAÇÃO NACIONAL DOS FARMACÊUTICOS, 2018).

Diante dos resultados é necessário promover a qualificação dos profissionais, haja vista que a maioria dos farmacêuticos $(80 \%)$ concluiu a graduação com currículos baseados nas DCN 2002. Embora tenham especialização e experiência em área clínica, a formação não reflete sua atuação, que pode ser decorrente da falta de clareza de suas atribuiçóes no Nasf e, consequentemente, de seu papel na APS.

Neste aspecto, a OPAS sinaliza que a formação prática do farmacêutico orientada mais ao produto do que aos serviços de saúde, dificulta o trabalho em equipe e a integração com a comunidade, consistindo em um grande desafio a ser superado para mudança deste paradigma (OPAS, 2013). Aliado a isso, estáo as demandas dos serviços técnico-gerenciais, as quais, muitas vezes, se sobrepóem às atividades assistenciais (LUQUETTI et al., 2017; NAKAMURA; LEITE, 2016). Portanto, o farmacêutico deve redefinir seu papel com segurança e autonomia para pôr em prática o Cuidado Farmacêutico, fundamental para transformar a realidade da AF (OLIVEIRA; ALVES; RAMALHO-DE-OLIVEIRA, 2017).

Destacam-se, além das limitaçooes na formação, a insegurança e medo do farmacêutico para atuar fora da farmácia, a falta de habilidades e capacitação para 
novas atividades, além do não reconhecimento, por ele próprio, pelas Equipes da Saúde da Família (EqSF) e usuários do seu papel como cuidador, podem levá-los a se prenderem a ela como espaço e atividade, limitando o fazer e os resultados em saúde esperados pela proposição do Nasf (NAKAMURA; LEITE, 2016).

\section{Caracterizaçáo das atividades do farmacêutico na APS}

A prática farmacêutica no Nasf contempla as duas grandes áreas da AF: técnico-gerencial e técnico-assistencial, o que diferencia o farmacêutico das demais categorias que compóem as equipes do Nasf, as quais são eminentemente assistenciais. Entretanto, considerando fundamental o aspecto clínico da AF ressaltase a importância do desenvolvimento das atividades assistenciais nos CS (BRASIL, 2018; BELO HORIZONTE, 2011).

Nesse sentido, na SMSA-BH, as atividades assistenciais seguem a lógica do Nasf com atuação integrada do profissional, o que permite realizar discussóes de casos clínicos, atendimento compartilhado, visitas domiciliares, construção conjunta de projetos terapêuticos de forma que amplia e qualifica as intervençôes no território e na saúde de grupos populacionais. Os pacientes atendidos pelos farmacêuticos provêm de encaminhamentos das EqSF e NASF, frequentemente nas reunióes de matriciamento, quando são repassados casos novos e as devolutivas dos pacientes em AFT, compartilhando as informaçóes e o cuidado, ou em situações de demanda espontânea dos pacientes identificados no CS (BELO HORIZONTE, 2018). Em relação ao apoio matricial, Castro e Campos (2016) destacam que além do cuidado compartilhado, essa metodologia funciona, ao mesmo tempo, como uma forma de educação permanente, uma vez que os profissionais com distintas formaçóes ampliam a comunicação entre eles ao conduzirem casos conjuntamente.

No presente estudo, $74 \%$ dos farmacêuticos relataram possuir perfil predominantemente técnico-gerencial, 13\% assistencial e 13\% ter equilíbrio entre ambos. A proporção de tempo dedicada às atividades assistenciais foi de 37\%. Estes dados corroboram os resultados da Pesquisa Nacional sobre Acesso, Utilização e Uso Racional de Medicamentos - Serviços (PNAUM), em relação às atividades farmacêuticas de natureza clínica na APS no Brasil, onde se constatou que do total de farmacêuticos entrevistados pouco mais de 1/5 afirmou realizar alguma atividade de natureza clínica (ARAÚJO et al., 2017a). 
O desconhecimento do seu papel assistencial em outros espaços além da farmácia, distorcido pela demanda dos demais profissionais da saúde e da comunidade como sendo a garantia do acesso aos medicamentos, reflete na seguinte fala:

Diante das principais atividades desenvolvidas, sobressaem-se as atividades técnico-gerenciais, pois essas são as atividades mais cobradas e percebidas pela comunidade, EqSF e outros profissionais de saúde, em geral, cuja prioridade é garantir o acesso ao medicamento. [F25]

Entretanto, quando questionados sobre as atividades assistenciais que desempenham, a maioria relatou executar aquelas referentes ao cuidado farmacêutico (figura 1).

Figura 1. Atividades técnico-assistenciais relatadas pelos farmacêuticos da Atenção Primária à Saúde da Secretaria Municipal de Saúde de Belo Horizonte, 2016

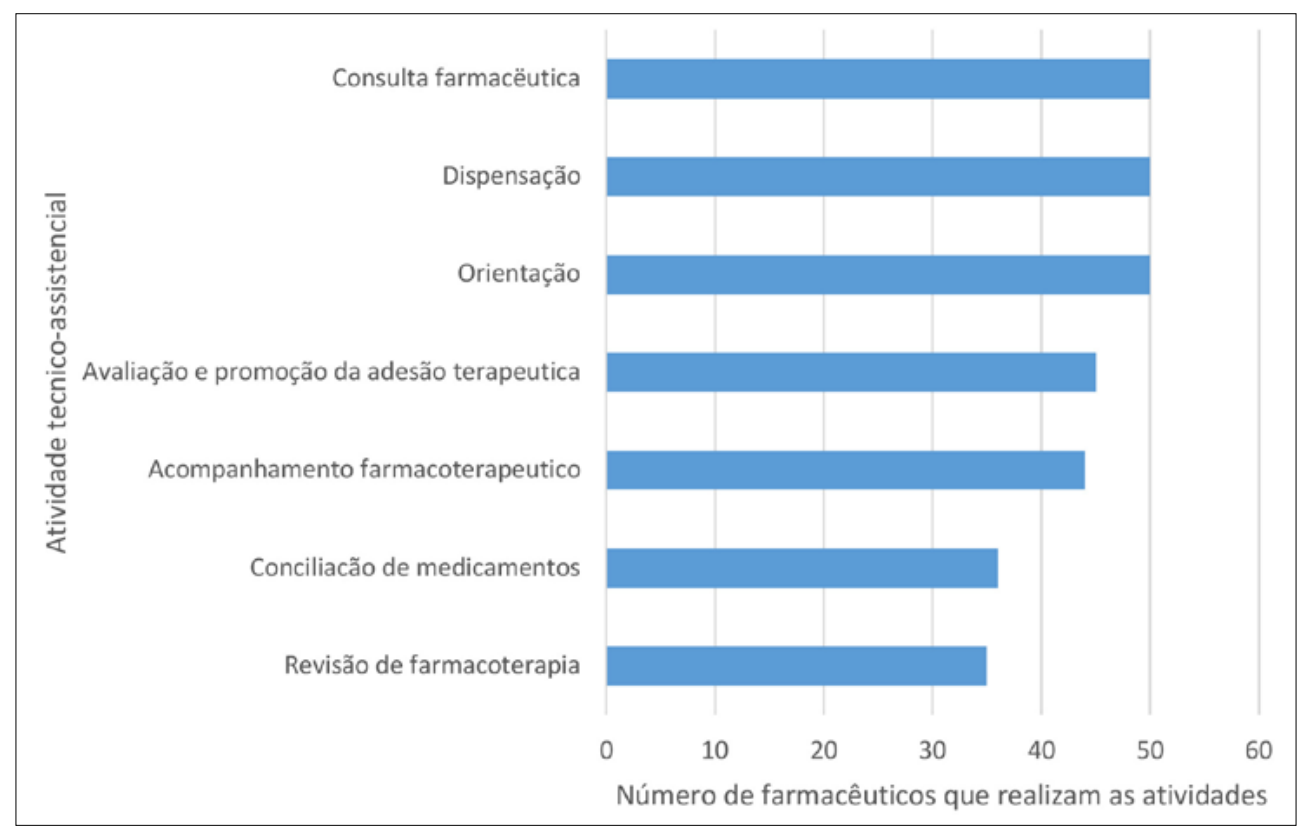

Fonte: Dados da pesquisa.

Todos relataram realizar consultas farmacêuticas com o usuário, dispensação e orientação farmacêutica. Ressalta-se que $88 \%$ assinalaram realizar o AFT, o que contrasta com o percentual de profissionais que citou usar algum método para tal referenciado na literatura científica (13\%). Entre os métodos mais usadas no Brasil estão Método Dáder, Pharmacotherapy Workup e Therapeutic Outcomes 
Monitoring (CORRER; OTUKI, 2013). Destaca-se na presente pesquisa que ter experiência em Atençáo Farmacêutica, seja ela formativa ou laboral, foi a única variável associada à aplicação de método de AFT $(\mathrm{p}<0,05)$.

Ademais, 98\% relataram registrar os atendimentos, $83 \%$ negociavam com o paciente a intervenção e $83 \%$ informaram contatar a equipe em caso de necessidade. Todos os profissionais afirmaram participar das reunióes de matriciamento do Nasf, espaços para a discussão de casos, organização e execução do trabalho integrado entre o Nasf e as EqSF (BRASIL, 2018). Além disso, 98\% disseram fazer consultas compartilhadas com outros membros da equipe de saúde e $92 \%$ afirmaram realizar atividades pedagógicas para promoção do URM (educação de pacientes).

Apesar de declararem realizar atividades assistenciais, percebe-se certo conflito do profissional entre as atividades desenvolvidas e a definição e planejamento de prioridades:

Considero desenvolver mais atividades técnico-gerenciais que assistenciais, uma vez que, penso não adiantar acompanhar um usuário que não está fazendo uso do medicamento, por estar em falta na Unidade de Saúde e ele náo tem dinheiro para comprar, que trabalhar com ações para evitar esta falta. Por fim, penso que não adianta acompanhar uns poucos pacientes, enquanto muitas açóes gerenciais ainda são deficientes e ao resolvê-las pode-se beneficiar uma maior parcela da população. [F32]

Ao mesmo tempo, outros conseguem realizar ambas as atividades, apesar das dificuldades:

Eu me considero como profissional que desempenha as duas tarefas. Eu busco me qualificar e me envolver em atividades assistenciais tanto individual, quanto em grupo. Entretanto, é muito difícil dividir minha agenda com as atividades gerenciais. Nas atividades assistenciais, a falta de consultório e de carro para fazer visita domiciliar, tem interferido na quantidade de atendimentos. [F6]

O sistema está organizado com foco no acesso ao medicamento desvinculado de um serviço que garanta seu uso correto e seguro (SOARES et al., 2016). O predomínio dessas atividades é uma prática frequente na realidade do farmacêutico, mas é importante correlacioná-la às atividades clínicas de modo a contemplar todas as etapas que evolvem o medicamento, desde sua seleção até o monitoramento, sendo que todas atuam como engrenagens e não podem ser trabalhadas de forma isolada (BRASIL, 2018). Assim, para garantir a integralidade do tratamento medicamentoso, os farmacêuticos devem investir em serviços que deem conta das necessidades em saúde da população (FOPPA; CHEMELLO; FARIAS, 2016). 
Como principais limitações para as ações assistenciais, identificaram-se a indisponibilidade de tempo devido às demandas das EqSF e comunidade (resultados semelhantes aos de Luquetti et al. (2017) e Nakamura e Leite (2016), além da estrutura física do CS ou perfil do profissional. Resultados da PNAUM também evidenciaram que as atividades de natureza clínica desempenhadas pelos farmacêuticos ainda são incipientes. Os principias motivos alegados pelos farmacêuticos para não realizar tais atividades foram: nunca ter sido solicitado, não dispor de espaço físico e de tempo. Outros motivos citados incluem ausência de formação específica, falta de incentivo pelo município, ausência de estrutura física e pessoal insuficiente na farmácia (ARAÚJO et al., 2017a). Percebe-se que as contingências impostas pela rotina de trabalho nos CS o direcionam a um perfil predominantemente técnico-gerencial.

Contudo, um diferencial no cenário desta pesquisa são as estratégias adotadas para avançar no desenvolvimento das atividades assistenciais no contexto da APS:

Eu me considero de caráter técnico-gerencial, todavia as atividades assistenciais não deixam de ser realizadas [...] O seguimento farmacêutico é dificultado, mas damos muitas orientaçôes aos pacientes a todo momento. Todos os casos de tuberculose e hanseníase são acompanhados no momento, tenho dado atenção especial também aos usuários que utilizam insulina, com realizaçáo de grupos e atendimentos individuais sempre que possível. [E8].

Assim, faz-se necessário estabelecer prioridades para prover o Cuidado Farmacêutico e o serviço farmacêutico a ser desenvolvido de forma programada e sistematizada, considerando os determinantes para definição do método a ser utilizado.

\section{Determinantes para a provisão do acompanhamento farmacoterapêutico}

No processo de cuidado, o farmacêutico, em colaboraçáo com o indivíduo e outros membros da equipe de saúde, busca melhorar os resultados da farmacoterapia, a fim de garantir que os medicamentos utilizados pelo indivíduo sejam corretamente indicados, efetivos, seguros e convenientes. Ademais, objetiva a prevenção, detecção e resolução de problemas relacionados aos medicamentos (PRM) antes que estes deem lugar à morbidade e mortalidade associadas à farmacoterapia (BRASIL, 2018). Os PRM, por sua vez, são definidos como "um evento indesejado experienciado pelo paciente que envolve ou se suspeita envolver a farmacoterapia, interferindo no resultado esperado da terapia e que requer julgamento profissional para ser resolvido."(CIPOLLE; STRAND; MORLEY, 2012). 
Após identificação dos PRM durante a avaliação inicial, o plano de cuidado deve ser desenvolvido podendo as intervençóes ser pactuadas com as equipes ou paciente. Durante esse processo o próprio profissional estabelece o intervalo necessário entre as consultas para que as intervençóes possam ser avaliadas (BRASIL, 2018).

A falta de diretrizes e de formação profissional para a sistematização do Cuidado Farmacêutico na APS aliada à insegurança do profissional reflete na sua atuação:

Ainda é necessário um posicionamento mais direcionado pelo farmacêutico do Nasf em relação a sua contribuição para o cuidado do paciente. Penso que os farmacêuticos ainda náo se sentem seguros ou situados dentro desse processo. [F35]

Seria necessária a padronização de condutas, confecção de protocolos, para dar embasamento e orientar o atendimento. Me sinto insegura ainda em fazer um acompanhamento farmacoterapêutico. [F43]

Em Belo Horizonte, com a implantação dos Nasf em 2008, foi priorizada a relação de um farmacêutico por equipe, que referencia em média 2,4 CS, com $56 \%$ deles por até dois CS, conforme cobertura das EqSF, entretanto, constatou-se que isto ainda é insuficiente. As atribuiçóes do farmacêutico na APS extrapolam os encaminhamentos do Nasf, o que o diferencia dos demais profissionais, conforme destaque anterior. Portanto, é imperativo estabelecer prioridades para o desenvolvimento das atividades assistenciais ao definir a agenda, atendendo às necessidades da população, interligando-as com as gerenciais com foco no paciente:

Todas as ações desenvolvidas têm como foco principal o paciente. Mesmo as atividades gerenciais que ocupam majoritariamente a carga horária, são desenvolvidas de forma a prestar uma melhor assistência ao usuário. Dentro de todas as atividades desenvolvidas, a demanda do usuário sempre é priorizada em relaçâo às atividades gerenciais, que muitas vezes, são desenvolvidas nos intervalos entre atendimentos, reunióes de matriciamento, grupos, dentre outras. [F11]

E ainda apresentam estratégias para resolver a demanda das atividades técnicogerenciais:

Considero-as principalmente técnico-gerencias visto que supervisiono ou melhor, coordeno três farmácias grandes, população acima de 100.000 habitantes. $\mathrm{O}$ treinamento constante de pessoal e assédio de outros profissionais no que concerne aos medicamentos disponíveis na rede, é muito grande. No entanto, estou tentando aumentar a proporção de \% assistencial, através de uma melhor organização de minhas açôes junto ao Nasf, com estudos de demanda e marcação de atendimentos prioritários. [E31]

Outro profissional considera suas açóes predominantemente assistenciais com destaque para as oportunidades que surgem na APS: 
Caráter eminentemente assistencial. Identifico uma demanda muito grande para trabalhar em nível assistencial, seja de forma coletiva ou individual. Entendo que a inserção do farmacêutico é primordial e ela não acontecerá ou será "legitimizada" sem esse trabalho de impacto e aproveitamento de oportunidades. [F24]

Aceitar seu papel social e adotar o cuidado como sua missão profissional é, sem dúvida, uma das grandes questóes que movimenta as discussôes da profissão farmacêutica a nível mundial nas últimas décadas (COSTA et al., 2017b; CFF, 2016; FREITAS; RAMALHO-DE-OLIVEIRA; PERINI, 2006).

No Brasil, há um considerável crescimento da implantação de serviços clínicos, sejam a nível hospitalar, ambulatorial ou na APS, públicos ou privados. A atuação do farmacêutico no cuidado direto ao IFC é um desafio para o sistema de saúde brasileiro (CFF, 2016). Destacam-se experiências exitosas em alguns municípios brasileiros que implantaram o serviço de Cuidado Farmacêutico, mostrando suas dificuldades e frutos que estão colhendo atualmente - Curitiba, Salto Grande, Divinópolis, São Paulo, Belo Horizonte (BRASIL, 2014; BRASIL, 2019).

Quanto ao cenário externo, Reino Unido, Austrália, Nova Zelândia, Espanha, Portugal, Holanda, Suíça e Estados Unidos da América implantaram serviços farmacêuticos na APS e incentivaram a ampliação da atuação clínica do farmacêutico como estratégia para a obtenção dos melhores resultados com os tratamentos e outras tecnologias em saúde, e obtiveram efeitos positivos (INTERNATIONAL PHARMACEUTICAL FEDERATION, 2011; CHEN, 2016; NEW ZEALAND MINISTRY OF HEALTH, 2016; CFF, 2016; COSTA et al., 2017b; NHS ENGLAND, 2019. CLINICAL PHARMACISTS IN GENERAL PRACTICE, 2019; BRASIL, 2019).

Nesta pesquisa o foco foi compreender como o AFT vem sendo realizado na APS em Belo Horizonte. Pelos relatos dos farmacêuticos que citaram usar um método de AFT referenciado na literatura, constatou-se um fluxo de trabalho melhor definido na unidade de saúde, e o estabelecimento de parcerias com outros profissionais:

Após passar dois anos na residência multiprofissional Saúde dos Idosos - Hospital das Clínicas ampliei o olhar e ação no campo técnico-assistencial. Atualmente empodero os trabalhadores da farmácia a assumirem as atividades técnico-gerenciais, tendo como referência minha gestão. E pactuo com a Equipe de Saúde da Família o desenvolvimento da farmácia clínica na unidade. [F18]

É importante ter um fluxo claramente definido, identificando quais pacientes podem ser de fato beneficiados pelo serviço. Podem existir duas "portas" para o 
AFT na APS: Indivíduos para os quais as equipes do CS identificam necessidade de atendimento farmacêutico e indivíduos que são identificados na farmácia do CS durante a aquisição de seus medicamentos e que demonstram estar confusos com a farmacoterapia (BRASIL, 2018).

Destaca-se que a maioria dos farmacêuticos (90\%) afirmaram agendar consultas de retorno com os pacientes. Isso afirma a continuidade do cuidado provido pelo farmacêutico em múltiplas consultas, tornando possível que muitos dos serviços apresentados (educação em saúde, rastreamento em saúde, conciliação de medicamentos e revisão da farmacoterapia) possam ser realizados durante o processo de acompanhamento do paciente:

O retorno é agendado de acordo com a minha avaliação da necessidade do paciente, para verificar o resultado de uma intervenção, ou de um parâmetro de monitorização; não tem uma frequência padronizada." [E6]

Depende da demanda do paciente, se maior agenda para um mês; se média dois meses e se baixa, faço monitoramento do paciente de seis em seis meses. [E7]

Os motivos apontados para não realização de consultas de retorno foram relacionados à disponibilidade de consultórios ou carro para visitas domiciliares, desejo do paciente, ausência de cuidador para receber as orientaçôes, no caso de impossibilidade por parte do paciente:

$\mathrm{O}$ agendamento pode ser feito diretamente com o próprio paciente/família ou por meio das reuniōes de matriciamento. A frequência do agendamento ocorre conforme a necessidade do caso, podendo ser semanal, quinzenal, mensal, bem como trimestral em alguns casos. Nem sempre é possível agendar no prazo ideal, devido ao número de consultórios insuficiente para organizaçáo do mapa de salas das unidades de saúde, o que limita o número de vagas. [E21]

Em geral, os agendamentos são realizados em reunião da equipe, após devolutiva de casos. Os retornos de atendimentos individuais são realizados de acordo com a demanda e disponibilidade de consultório para atendimento, sendo que a disponibilidade de consultório é um dos motivos que eventualmente pode impedir o agendamento. [E25]

Os resultados apontados pela PNAUM também indicam a necessidade de estruturação da ambiência dos serviços de farmácia visando à humanização do atendimento ofertado e a melhoria das condiçóes de trabalho aos profissionais (LEITE et al., 2017). De forma complementar para a implementação do cuidado farmacêutico é necessária colaboração dos demais profissionais e conhecimento dos usuários sobre o serviço (OLIVEIRA; ALVES; RAMALHO-DE-OLIVEIRA, 
2017). O Nasf é relativamente novo no SUS e os profissionais não foram formados para utilizar tecnologias leves, o que corrobora para o desconhecimento do papel do farmacêutico pelas EqSF e Nasf, refletindo no sucesso do serviço farmacêutico (NAKAMURA; LEITE, 2016):

Mas tenho poucos pacientes acompanhados devido ao baixo número de encaminhamentos. [F23]

Por estar em processo de construção, é possível observar sua dinâmica na transformação do profissional e seu reconhecimento perante à EqSF:

A minha realidade atual de trabalho apresenta predominância técnico-gerenciais [...] Acredito que o perfil das três unidades nas quais trabalho, unidades de baixo risco, com usuários questionadores e elevada demanda de questôes técnicas, resultam nesse perfil. No entanto, aos poucos, a tentativa está sendo de vínculo maior com a equipe de saúde em atividades assistenciais. A medida que as intervenções e acompanhamentos realizados pela farmacêutica apresentam bons resultados e os outros profissionais reconhecem o papel e importância do farmacêutico os encaminhamentos aumentam. [F17].

Apresentam-se, portanto, situaçóes distintas pelos relatos apresentados: farmacêuticos que não recebem encaminhamentos e outros com uma demanda reprimida de pacientes que necessitam do AFT.

A maioria dos farmacêuticos relatou realizar AFT, mas poucos adotam um método referenciado na literatura, condição essencial para caracterizar esse serviço, configurando uma contradição. A condução das consultas farmacêuticas mostra que as atividades se encontram pulverizadas e o cuidado fragmentado, sinalizando para a necessidade de sua sistematização, para obtenção de resultados mensuráveis, conforme evidenciado no seguinte relato:

Atendo dúvidas, faço dispensaçôes de medicamentos. Elaboro esquema terapêutico com avaliaçấo da receita. Entrega de caixas com divisórias para o período de manhã, tarde e noite; ou construção de envelopes com todos os medicamentos fracionados nos três períodos do dia. Oriento e faço arguiçáo para o paciente ou seus familiares. Marco retorno e avalio evoluçâo. Estudo possíveis interaçôes. Procuro a equipe e informo irregularidades; retiro medicamentos vencidos e organizo estoques, ainda náo consegui adotar um método de acompanhamento farmacoterapêutico. [F31]

A fala anterior corrobora com uma pesquisa realizada sobre a organização dos serviços farmacêuticos clínicos no SUS (ARAÚJO et al., 2017b). Eles observaram que em todas as regiōes administrativas do país esses serviços não são realizados de forma consistente, evidenciando as dificuldades no desenvolvimento dessa prática 
profissional no país. Espera-se que Belo Horizonte possa servir de modelo aos municípios em que o farmacêutico ainda não está inserido no Nasf.

É evidente que o farmacêutico do Nasf acumula múltiplas funções, portanto, o mesmo deve reservar carga horária para o atendimento clínico. O farmacêutico precisa se transformar de um profissional técnico para um cuidador, e sistematizar sua prática no contexto do Nasf (SILVA et al., 2016).

Relatos da presente pesquisa apontam para dificuldades estruturais e processuais do sistema de saúde. A esse respeito ressalta-se a necessidade de investimentos e organizaçáo do serviço, mudanças no processo de trabalho do farmacêutico, na farmácia, o que constitui premissa para o desenvolvimento da clínica (SOARES et al., 2016). Araújo et al. (2017a) consideram que apesar de importantes políticas farmacêuticas no Brasil tenham colocado o Cuidado Farmacêutico como diretriz, é fundamental empreender esforços no sentido de institucionalizar essas atividades clínicas, assegurando estrutura adequada, qualificação dos profissionais, financiamento e avaliação dos resultados para que farmacêuticos e gestores sejam estimulados a oferta-las no SUS promovendo a implementação da Política Nacional de Assistência Farmacêutica em todas as suas diretrizes.

Cabe ressaltar, que vivenciamos um processo de trabalho em construção em todo o SUS, pois nossos dados corroboram com outras experiências, ao retratarem dificuldades semelhantes (SILVA et al., 2012; BRASIL, 2015; CFF, 2015; SILVA, 2016; FREITAS et al., 2016; NAKAMURA; LEITE, 2016; SOARES et al., 2016; ARAÚJO et al., 2017a; LUQUETTI et al., 2017). Contudo, estudos reforçam que a sistematização do Cuidado Farmacêutico na APS traz benefícios e impacta na qualidade de vida dos pacientes, bem como reduz custos para o sistema de saúde, destacando sua importância nos níveis social e econômico (CFF, 2015; BRASIL, 2014; SOARES et al., 2016; FOPPA; CHEMELLO; FARIAS, 2016; BRASIL, 2015; MENDONÇA et al., 2016; MESSERLI et al., 2016; OBRELI-NETO, 2015; SABATER-HERNÁNDEZ et al., 2016).

Tendo em vista a amplitude de açóes que podem ser desenvolvidas por esse profissional, torna-se importante contextualizar os possíveis cenários de prática dentro da APS (BRASIL, 2018), contribuindo para efetividade dos serviços oferecidos à população. A implementação desse serviço requer reflexão e construção coletivas locais, não existe modelo validado, portanto, necessita realimentar-se de experiências externas (OPAS, 2013). Nesse sentido, destacam-se no cenário nacional, 
esforços direcionados para a sistematização do Cuidado Farmacêutico no SUS, que contribuem para identificar potencialidades e desafios enfrentados no cotidiano de trabalho (NAKAMURA; LEITE, 2016; SILVA, 2016; BRASIL, 2015; OLIVEIRA et al., 2015; PEREIRA; LUIZA; MARQUES-DA-CRUZ, 2015; SILVA et al., 2018).

Cabe reconhecer o papel do profissional farmacêutico como protagonista, indutor e qualificador dessas práticas na APS. Assim, quando presente, o farmacêutico visa qualificar o acesso da população à farmacoterapia, contribui para o URM, além de proporcionar cuidado farmacêutico aos usuários e à comunidade (BRASIL, 2018). Destaca-se que a APS representa uma excelente oportunidade para reorientar os serviços farmacêuticos como parte integral dos serviços de saúde (OPAS, 2013; RAMALHO-DE-OLIVEIRA, 2011).

Diante do panorama mundial da profissão relacionado ao Cuidado Farmacêutico na APS, conforme apresentado anteriormente, a SMSA-BH tem adotado diversas estratégias. Várias atividades relacionadas à gestão técnica do medicamento já se encontram consolidadas no município, sendo descritas em manuais e procedimentos operacionais padrão. O desenvolvimento das atividades clínicas ainda é um processo em construção, para o qual, em 2016, em parceria com o MS foi feito um diagnóstico da atuação do farmacêutico centrada no paciente, cujo objetivo foi implantar açóes que sistematizem o Cuidado Farmacêutico, definindo diretrizes e estratégias necessárias para desenvolvê-lo (BELO HORIZONTE, 2016).

Neste estudo, os farmacêuticos relataram realizar consultas, mas não tínhamos como avaliar se ao afirmarem isto, estavam se referenciando ao AFT, o que caracteriza uma limitação. Entretanto, atualmente, tem-se os indicadores de produção gerencial e assistencial dos farmacêuticos, por meio do Software Gerenciamento da Assistência Farmacêutica (GERAF) desenvolvido para monitorar e avaliar as açóes (BELO HORIZONTE, 2017). Por este recurso será possível quantificar os serviços farmacêuticos efetivamente realizados pelos profissionais, registrados em prontuário eletrônico, contribuindo para sua qualificação e sistematização.

Cabe ressaltar que no Brasil a carência de consenso sobre conceitos, métodos e competências para o Cuidado Farmacêutico resulta na sua fragmentação, reduzindo o impacto da prática e inibindo o desenvolvimento de estratégias de ação mais efetivas (CFF, 2016; FREITAS; RAMALHO-DE-OLIVEIRA; PERINI, 2006), isso pode ser considerado como uma limitação da presente pesquisa. Neste sentido, em maio de 2018, foi publicado o documento que define as Diretrizes para a AF 
Integral em Belo Horizonte, com o propósito de estabelecer a uniformização de conceitos, a sistematização das ações desenvolvidas pelos farmacêuticos em todos os níveis de atenção e a definição de indicadores de monitoramento e avaliação da AF na SMSA/BH (BELO HORIZONTE, 2018).

\section{Considerações finais}

Este estudo permitiu fazer um diagnóstico da atuação farmacêutica, fundamental para direcionar a sistematização do Cuidado Farmacêutico e sua consolidação no sistema de saúde, considerando as possibilidades de transformação do farmacêutico através da reflexão de sua prática.

Evidenciam-se novas exigências em relação à profissão farmacêutica no SUS. Para tanto, são necessários investimentos para reorganizar os processos de trabalho, qualificar os trabalhadores (com ações de educação permanente e continuada) e reestruturar a agenda conforme diretrizes institucionais, visando à ampliação do acesso aos serviços farmacêuticos destinados ao IFC, com suas necessidades de saúde, contribuindo de forma efetiva para mudança do foco, anteriormente centrado no medicamento.

Por fim, observou-se que o Cuidado Farmacêutico é realidade na APS, porém, apesar de prioritário, constitui, ainda, um desafio para os farmacêuticos, principalmente devido à demanda de atividades gerenciais, à deficiência na formação para o cuidado e falta de clareza de seu papel no cuidado ao paciente. Contudo, são notórios os avanços para a organizaçáo do serviço no contexto multiprofissional e interdisciplinar centrado no usuário, fundamentais para atingirmos um dos objetivos da profissão: o Cuidado Farmacêutico. ${ }^{1}$

\section{Referências}

ARAÚJO, P. S. et al. Atividades farmacêuticas de natureza clínica na atenção básica no Brasil. Revista de Saúde Pública, v. 51, supl. 2, p. 6s, 2017a. Disponível em: https:/doi.org/10.11606/ S1518-8787.2017051007109.

ARAÚJO, S. Q. et al. Organização dos serviços farmacêuticos no Sistema Único de Saúde em regiōes de saúde. Ciência \& Saúde Coletiva, Rio de Janeiro, v. 22, n. 4, p. 1181-1191, abr. 2017b. Disponível em: http://dx.doi.org/10.1590/1413-81232017224.27042016. Acesso em: 20 nov. 2018. 
BARBERATO, L. C.; SCHERER, M. D. A.; LACOURT, R. M. C. O farmacêutico na atenção primária no brasil: uma inserção em construção. Ciência \& Saúde Coletiva. Disponível em:http://www.cienciaesaudecoletiva.com.br/artigos/o-farmaceutico-na-atencao-primaria-nobrasil-uma-insercao-em-construcao/16679?id=16679. Acesso em: 5 mar. 2019.

BARDIN, L. Análise de conteúdo. São Paulo: Ediçôes 70, 2016.

BELO HORIZONTE. Secretaria Municipal de Saúde. Gerência de Medicamentos. Projeto: Assistência Farmacêutica para atençâo primária em Belo Horizonte. Belo Horizonte: SMSA/ GEMED, 2011.

. Gerência de Assistência Farmacêutica (GEAF). II Seminário de Assistência Farmacêutica da SMSA-BH. Belo Horizonte: SMSA/GEAF, set. 2016.

- Gerência de Assistência Farmacêutica. Manual Técnico Operacional do Software GERAF "Gerenciamento da Assistência Farmacêutica”. Orientaçóes Técnicas Farmácias Unidades Básicas de Saúde. Belo Horizonte: SMSA/GEAF, 2017.

. Gerência de Assistência Farmacêutica. Diretrizes para a Assistência Farmacêutica Integral em Belo Horizonte. Belo Horizonte: SMSA/GEASF, 2018.

BRASIL. Ministério da Educação. Conselho Nacional de Educação. Câmara de Educação Superior. Resolução CNE/CES 2, de 19 de fevereiro de 2002. Institui Diretrizes Curriculares Nacionais do Curso de Graduação em Farmácia. Diário Oficial da União. Brasília, 4 mar. 2002. Seção 1, p. 9.

- Conselho Nacional de Educação, Câmara de Educação Superior (BR). Resolução CNE/CES 6/2017. Institui as Diretrizes Curriculares Nacionais para o Curso de Graduação em Farmácia. Diário Oficial da União, Brasília, 20 out. 2017. Seção 1, p. 30.

- Ministério da Saúde. Secretaria de Ciência, Tecnologia e Insumos Estratégicos. Departamento de Assistência Farmacêutica e Insumos Estratégicos. Serviços farmacêuticos na atenção básica à saúde. Brasília: Ministério da Saúde. 2014 (Cuidado farmacêutico na atenção básica; caderno 1).

- Secretaria de Ciência, Tecnologia e Insumos Estratégicos. Departamento de Assistência Farmacêutica Insumos Estratégicos. Resultados do projeto de implantação do cuidado farmacêutico no Município de Curitiba. Brasília: Ministério da Saúde, 2015. 100 p. (Cuidado farmacêutico na atenção básica; caderno 4).

- Secretaria de Atenção à Saúde. Departamento de Atenção Básica. Práticas Farmacêuticas no Núcleo de Apoio à Saúde da Família (Nasf). Brasília: Ministério da Saúde, 2018. 33p. Disponível em: http://bvsms.saude.gov.br/bvs/publicacoes/praticas_farmaceuticas_ apoio_saude_familia. Acesso em: 24 out. 2018. 
- Programa de Apoio Desenvolvimento Institucional do Sistema Único de Saúde PROADI/SUS. Projeto Atenção Básica Capacitação, Qualificação dos Serviços de Assistência Farmacêutica e Integração das Práticas de Cuidado na Equipe de Saúde. Desenvolvimento dos serviços de Cuidado Farmacêutico. Ebook 1; 2019.

CASTRO, C. P.; CAMPOS, G. W. S. Apoio Matricial como articulador das relaçóes interprofissionais entre serviços especializados e atenção primária à saúde. Physis, Rio de Janeiro, v. 26, n. 2, p. 455-481, jun. 2016. Disponível em: http://dx.doi.org/10.1590/S010373312016000200007. Acesso em: 13 mar. 2019.

CASTro, M. S. A Formação do Farmacêutico para a Atenção Primária à Saúde. IV Fórum Nacional de Educação Farmacêutica. ABENFAR, 2011. Disponível em: www.paho.org/bra/ images/stories/BRA02C/formacao_2.pdf. Arquivo PDF. Acesso em: 23 out. 2018.

CHEN, T. F. Pharmacist-Led Home Medicines Review and Residential Medication Management Review: The Australian Model. Drugs Aging, v. 33, n. 3, p.: 199-204, 2016 Mar. Disponível em: https://www.ncbi.nlm.nih.gov/pubmed/26961696. Acesso em: 30 jul. 2019.

CIPOLlE, R. J.; STRAND, L. M.; MORLEY, P. C. Pharmaceutical Care as a Professional Practice for Patient-Centered Medication Management Services. In: MGH Medical, ed. Pharmaceutical Care Practice - The Patient-Centered Approach to Medication Management Services. 3ed. Minnesota, p. 37-72, 2012.

CONSELHO FEDERAL DE FARMÁCIA. Perfil do farmacêutico no Brasil: relatório. Brasília: CFF, 2015.

- Serviços farmacêuticos diretamente destinados ao paciente, à família e à comunidade: contextualização e arcabouço conceitual. Brasília: Conselho Federal de Farmácia; 2016. 200 p. Disponível em: http://www.cff.org.br/userfiles/ProfarArcaboucoTELAFINAL.pdf. Acesso em: 20 dez. 2018.

CORRER, C. J.; OTUKI, M.F.; SOLER, O. Assistência farmacêutica integrada ao processo de cuidado em saúde: gestão clínica do medicamento. Revista Pan-Amazônica de Saúde, Ananindeua, v. 2, n. 3, p. 41-49, 2011.

CORRER, C. J.; OTUKI, M. F. A prática farmacêutica na farmácia comunitária. Porto Alegre: Artmed, 2013.

COSTA, K. S. et al. Avanços e desafios da assistência farmacêutica na atenção primária no Sistema Único de Saúde. Revista de Saúde Pública, São Paulo, v. 51, supl. 2, 3s, 2017a. Disponível em: http://dx.doi.org/10.11606/s1518-8787.2017051007146. Acesso em: 19 dez. 2019.

COSTA, F. A. et al. Provision of pharmaceutical care by community pharmacists across Europe: is it developing and spreading? Journal of Evaluation in Clinical Practice, v. 23, n. p: 1336-1347, 2017b. Epub 2017 Aug 1. Disponível em: https://www.ncbi.nlm.nih.gov/pubmed/28762651. Acesso em: 1 ago. 2019. 
FEDERAÇÃO NACIONAL DOS FARMACÊUTICOS. CNE aprova novas DCN do Curso de Graduação em Farmácia. Disponível em: http://fenafar.org.br/index.php/201601-26-09-32-20/fsa/1608-cne-aprova-novas-dcns-do-curso-de-graduacao-em-farmacia. Acesso em: 20 nov. 2018.

FOPPA, A. A.; CHEMELLO, C.; FARIAS, M. R. Caracterização Farmacoepidemiológica de Indivíduos com Doença de Parkinson para Implantação de Serviço Clínico Farmacêutico. Journal of Applied Pharmaceutical Sciences - JAPHAC, v. 3, n. 1, p. 28-40, 2016.

FREITAS, E. L.; RAMALHO-DE-OLIVEIRA D.; PERINI E. Atenção Farmacêutica Teoria e Prática: um Diálogo Possível? Acta Farmacéutica Bonaerense, Buenos Aires, v. 25, n. 3, p. 447-53, 2006.

FREITAS, G. R. M. et al. Principais dificuldades enfrentadas por farmacêuticos para exercerem suas atribuições clínicas no Brasil. Revista Brasileira de Farmácia Hospitalar e Serviços de Saúde, São Paulo, v. 7, n. 3, p. 35-41, jul-set. 2016.

. WORLD HEALTH ORGANIZATION. Annex 8: Joint FIP/WHO guidelines on good pharmacy practice: standards for quality of pharmacy services. The Hague: World Health Organization, 2011. 20p. Disponível em: http://apps.who.int/medicinedocs/documents/ s18676en/s18676en.pdf. Acesso em: 30 jul. 2019.

LEITE, S. N. et al. Infraestrutura das farmácias da atenção básica no Sistema Único de Saúde: Análise dos dados da PNAUM-Serviços. Revista de Saúde Pública, São Paulo, v. 51, supl. 2, 13s, 2017. Disponível em: http://dx.doi.org/10.11606/s1518-8787.2017051007120. Acesso em: 19 dez. 2019.

LUQUETTI, T. M. et al. Serviços farmacêuticos na atenção primária à saúde: Percepção dos farmacêuticos. DIVERSITATES International Journal, v. 9, n. 3, p. 27-43, 2017. Disponível em: www.diversitates.uff.br/index.php/1diversitates-uff1/article/download/229/128. Acesso em: 30 jul. 2019.

MENDONÇA, S. A. M. et al. Clinical outcomes of medication therapy management services in primary health care. Brazilian Journal of Pharmaceutical Sciences, v. 52, n. 3, p. 365-373, Sep. 2016. Disponível em: http://dx.doi.org/10.1590/s1984-82502016000300002. Acesso: 12 set. 2018.

MESSERLI, M. et al. Impact of a community pharmacist-led medication review on medicines use in patients on polypharmacy - a prospective randomised controlled trial. BMC Health Services Research, v. 16, n. 1, dec. 2016. Available from: http://bmchealthservres.biomedcentral. com/articles/10.1186/s12913-016-1384-8. Acesso: 11 set. 2018.

MINAYO, M. C. S. O desafio do conhecimento. Pesquisa qualitativa em saúde. 14 ed. São Paulo/ Rio de Janeiro: Hucitec/Abrasco, 2014. 
NAKAMURA, C. A.; LEITE, S. N. A construção do processo de trabalho no Núcleo de Apoio à Saúde da Família: a experiência dos farmacêuticos em um município do sul do Brasil. Ciência \& Saúde Coletiva [Internet], v. 21, n. 5, p. 1565-1572, mai. 2016. Disponível em: http://dx.doi. org/10.1590/1413-81232015215.17412014. Acesso em: 12 nov. 2018.

NEW ZEALAND MINISTRY OF HEALTH. Pharmacy Action Plan: 2016 to 2020. 2016. Disponível em: https://www.health.govt.nz/system/files/documents/publications/pharmacyaction-plan-2016-to-2020.pdf. Acesso em; 30 jul. 2019.

NHS ENGLAND. Clinical Pharmacists in General Practice [2019]. Disponível em: https:// www.england.nhs.uk/gp/gpfv/workforce/building-the-general-practice-workforce/cp-gp/. Acesso em: 30 jul. 2019.

OBRELI-NETO, P. R. et al. Economic evaluation of a pharmaceutical care program for elderly diabetic and hypertensive patients in primary health care: a 36-month randomized controlled clinical trial. Journal of Managed Care \& Specialty Pharmacy, v. 21, n. 1, p: 6675, 2015. Disponível em: http://www.ncbi.nlm.nih.gov/pubmed/25562774. Acesso em: 26 ago. 2018.

OLIVEIRA, G. C. B. et al. Reestruturação da assistência farmacêutica no município de Lagoa Santa (MG). Experiências exitosas de farmacêuticos no SUS. Conselho Federal de Farmácia, Brasília, v. 3, n. 3, p. 71-77, jul. 2015. Disponível em: http://www.cff.org.br/userfiles/file/ experi\%C3\%AAncias\%20exitosas\%202015_web.pdf. Acesso em: 16 out. 2018.

OLIVEIRA, G. C. B.; ALVES, M. R.; RAMALHO-DE-OLIVEIRA, D. Action research as a tool for transformation of the pharmacist's praxis in primary care. International Journal of Pharmacy and Pharmaceutical Sciences. v. 9, p. 180-185, 2017.

ORGANIZACIÓN PANAMERICANA DE LA SALUD. Servicios farmacéuticos basados en la atención primaria de salud. Documento de posición de la OPS/OMS. Washington, DC: OPS, 2013. (La Renovación de la Atención Primaria de Salud en las Américas, n. 6). Disponível em: http://www.paho.org/hq/index.php?option=com_docman\&task=doc_view\&gid=21582\&Item id=270\&lang=en. Acesso em: 20 out. 2018.

PEREIRA, N.C.; LUIZA, V. L.; MARQUES-DA-CRUZ, M. Serviços farmacêuticos na atenção primária no município do Rio de Janeiro: um estudo de avaliabilidade. Saúde debate, Rio de Janeiro, v. 39, n. 105, p. 451-468, abr-jun. 2015.

RAMALHO-DE-OLIVEIRA, D. Atençâo Farmacêutica: da filosofia ao gerenciamento da terapia medicamentosa. 1. ed. Sáo Paulo: RCN Comercial e Editora, 2011. 344p.

SABATER-HERNÁNDEZ, D. et al. A Systematic Review of Evidence-Based Community Pharmacy Services Aimed at the Prevention of Cardiovascular Disease. Journal of Managed Care \& Specialty Pharmacy, v. 22, n. 6, p. 699-713, 2016. 
SILVA, D. A. M. Gerenciamento da terapia medicamentosa na atenção primária do município de Belo Horizonte: uma autoetnografia da transformação profissional. 2016. 97 f. Dissertação (Mestrado em Medicamentos e Assistência Farmacêutica) - Faculdade de Farmácia, Universidade Federal de Minas Gerais, Belo Horizonte, 2016.

SILVA, A. T. C. et al. Núcleos de Apoio à Saúde da Família: desafios e potencialidades na visão dos profissionais da Atenção Primária do Município de São Paulo, Brasil. Cadernos de Saúde Pública, v. 28, n. 11, p. 2076-2084, 2012. Disponível em: http://dx.doi.org/10.1590/S0102311X2012001100007. Acesso em 22 out. 2018.

SILVA, D. A. M. et al. A prática clínica do farmacêutico no núcleo de apoio à saúde a família. Trabalho, Educação e Saúde, 2018. Disponível em: http://dx.doi.org/10.1590/1981-7746sol00108. Acesso em: 26 set. 2018.

SILVA, D. et al. A extensão universitária como caminho para a construção de serviço de gerenciamento da terapia medicamentosa na atenção primária à saúde. Revista Brasileira de Farmácia Hospitalar e Serviços de Saúde, São Paulo, v. 7, n. 2, p. 15-21, 2016.

SOARES L. et al. (Orgs.). Assistência Farmacêutica no Brasil: Política, Gestão e Clínica. Atuação clinica do farmacêutico. Florianópolis: Universidade Federal de Santa Catarina, 2016.

SOUSA, I. F.; BASTOS, P. R. H.; BOGO, D. Diretrizes curriculares nacionais: desafios na formação dos farmacêuticos para atuação no Sistema Único de Saúde. Revista Brasileira de Pesquisa em Saúde, Vitória, v. 15, n. 1, p. 129-134, 2013.

WIEDENMAYER, K. et al. Developing Pharmacy Practice. A focus on patient care. Geneva: WHO, 2006.

YIN, R. K. Estudo de caso: planejamento e métodos. 5 ed. Porto Alegre: Bookman, 2015.

\section{Nota}

${ }^{1}$ D. R. Destro, S. A. do Vale, M. J. M. Brito e C. Chemello: análise e interpretação dos dados; redação do artigo e revisão crítica relevante do conteúdo intelectual; Aprovação final da versão a ser publicada 


\section{Abstract}

\section{Challenges for pharmaceutical care in Primary Health Care}

This case study aimed to describe the profile of pharmacists, characterize the pharmaceutical services, and unveil the determinants for the provision of pharmacotherapeutic follow-up based on the model of practice of Pharmaceutical Care in Primary Health Care. Fifty pharmacists answered a questionnaire about their profile and activities. The Bardin content analysis was applied to analyze the data on the activities, resulting in two categories: characterization of the activities performed by pharmacists and the determinants for the provision of pharmacotherapeutic follow-up. It was observed that pharmaceutical care is still a challenge to be faced, mainly due to the demand for managerial activities and the deficiency in training for care, needing to reorganize work processes and institutional guidelines to expand access to patientcentered pharmaceutical services. However, the growing number of pharmacists working in care, reorganizing and redefining their role, seeking to transform the reality of the Municipal Pharmacy Assistance, stands out. Despite the still existing challenges related to training, structure and processes, the search for solutions for the organization of the service, with the improvement of the clinical activities, strengthening the Pharmaceutical Assistance in the Unified Health System, stands out.

Keywords: Pharmaceutical Care, Pharmaceutical Services, Primary Health Care, Unified Health System. 